\title{
Autoimmunity in hereditary retinal degenerations. II. Clinical studies: antiretinal antibodies and fluorescein angiogram findings
}

\author{
JOHN R HECKENLIVELY, ALFRED M SOLISH, SUSAN M CHANT, \\ AND ROBERTA H MEYERS-ELLIOTT
}

From the Jules Stein Eye Institute and the Department of Ophthalmology, UCLA School of Medicine, Los Angeles, CA 90024, USA

SUMmARY Testing by indirect immunofluorescence for the detection of antiretinal antibodies and lymphocyte stimulation for cell-mediated immunity to retinal antigens was performed on blood obtained from 59 patients with retinitis pigmentosa (RP) and 29 without RP who had other types of retinal disease. The results from the patients' immunological studies were correlated in a masked fashion with six parameters of the fluorescein angiogram: disc staining, peripapillary oedema, vascular arcade oedema, macular oedema, and focal vascular staining (late phases), and disc telangiectasia (early phases). Significant correlations for both groups together were found for IgG antiretinal antibody reactivity and macular oedema $(p<0.038)$ and disc staining $(p<0.033)$. The non-RP retinal disease group had more significant correlations, including IgG antiretinal antibody reactivity with vascular arcade oedema $(\mathrm{p}<0.018)$, disc staining $(\mathrm{p}<0.018)$, and peripapillary oedema $(\mathrm{p}<0.023)$; the RP patients had significant correlation with IgG reactivity and arcade oedema ( $\mathrm{p}<0.045)$. With combinations of IgG, IgM, and lymphocyte reactivity various significant. correlations were found with the fluorescein angiogram.

Numerous laboratory studies support the notion that a variety of acquired and hereditary retinal diseases have autoimmune components, and reports to date have emphasised the presence of cell-mediated immunity or antiretinal antibodies to various retinal antigens in these patients. Whether a pathological role is played by these antibodies or by lymphocytes showing sensitisation to retinal antigens has been more difficult to determine. Retinal tissue is rarely available for direct study, and indirect methods are usually employed to investigate the possibility of immunopathogenicity in the retinal degenerations.

Clinicopathological correlations have been attempted by several investigators. Dumonde et al. examined sera from patients with various forms of retinal vasculitis for the presence of complement and antiretinal antibodies.' In addition to identifying immunological activity they also attempted to correlate its degree with the severity of the systemic and

Correspondence to John R Heckenlively, MD, Jules Stcin Eyc Institutc, UCLA School of Medicinc, Los Angeles, CA 9(X)24, USA. retinal vasculitis. In patients with only ocular involvement retinal autoantibodies were found significantly more often those with severe retinal vasculitis than in those who had only mild to moderate disease.

Keltner $e t$ al. reported on a cancer patient with an unusual retinal degeneration whose serum antibodies were reactive with normal donor retinal tissue by the immunofluorescent complement fixation test. ${ }^{2}$

Numerous studies have established that patients with retinitis pigmentosa (RP) and other retinal degenerations frequently have an immunological response to retinal antigens. Alterations in cellmediated as well as humoral immunity have been described. Brinkman et al. $^{3}$ and Heredia et al. ${ }^{+}$ described retinal antigen stimulation of lymphocytes from RP patients, and Char $e$ t al. found that lymphocytes from RP patients were significantly more cytotoxic in an assay against a retinoblastoma tissue culture cell line. ${ }^{5}$ Increased cellular immune responsiveness to the retinal $S$ antigen has been found in RP patients ${ }^{6}$ as well as patients with retinal inflammatory diseases such as birdshot retinochoroidopathy ${ }^{7}$ and ${\text { uveitis. }{ }^{\text {}}}$ 
Hooks et al. recently reported subnormal levels of a lymphokine, interferon-gamma, a product of T lymphocytes from 12 patients with RP. No abnormality of humoral immunity or any increased incidence of infections was detected in these patients."

While Rahi reported increased serum IgM levels in RP patients," a similar study by Spiro et al. found no difference in serum IgM levels between RP patients and controls." Kumar et al. found antiretinal antibodies by the tanned red cell haemagglutination technique in $60 \%(15 / 25)$ of RP cases that they investigated. ${ }^{12}$ Spalton et al. using immunofluorescence techniques demonstrated the binding of sera from RP patients to rat photoreceptor cells. ${ }^{13}$ Recently our group reported that $37 \%$ (43/116) of RP patients and $33 \%(21 / 64)$ of non-RP patients with other retinal disease had antiretinal antibodies by indirect immunofluorescent techniques compared with $2 \%(1 / 42)$ of controls. ${ }^{1+}$ The same study showed that $19 \%(16 / 86)$ of RP patients and $18 \%(11 / 64)$ of non-RP patients with other retinal eye disease had sensitisation of lymphocytes to retinal antigens when tested with in-vitro transformation assays."

While all these studies strongly suggest that the immune system may have an active role in RP and other retinal degenerations, no study to date has demonstrated a clinical correlation between the presence of immunological response and an objective measure of the patient's disease state.

To ascertain whether antiretinal antibodies and sensitised lymphocytes may have a pathophysiological role in RP and other retinal degenerations, we correlated in a double-masked fashion the fluorescein angiographic findings with the presence or absence of antiretinal antibodies and cell-mediated immunity to retinal antigens in patients with RP and with non-RP ocular disease.

\section{Patients and methods}

Fifty-nine patients with all types of RP and 29 with other types of retinal disease, a subset of a larger group previously reported," were examined to see whether the presence of antiretinal immune activity correlated with findings on the fluorescein angiogram. Indirect immunofluorescence staining for IgG and IgM antiretinal antibodies and lymphocyte invitro transformation assays to retinal antigens were performed by methods previously reported. ${ }^{14}$ Patients selected for study were all those in whom a fluorescein angiogram was obtained as part of their routine diagnostic investigation within two months of venepuncture for the immunological studies. In most cases the fluorescein angiogram was obtained on the same day as the venepuncture was performed, but a few patients had fluorescein angiograms performed on return visits or a few days after the initial visit if an appointment was not available the same day. Selection was not based on any knowledge of the blood results.

Patients with progressive panretinal degeneration and other ocular disorders were evaluated with a comprehensive medical, ocular, and family history (pedigree analysis), ophthalmic examination, and tests consisting of Goldmann visual fields, electroretinogram, dark adaptation final rod threshold, fundus photography, and fluorescein angiography. Patients with hereditary retinal degenerations were classified as precisely as possible by the above tests and examination. RP was diagnosed when the patient had a panretinal degeneration with progressive visual field loss, and an abnormal or unrecordable electroretinogram with no other inflammatory or toxic history to explain the retinal degeneration.

The patients were placed in the following RP subgroups: rod-cone and cone-rod degeneration subdivided by inheritance, and the specific RP types of choroideraemia, preserved para-arteriolar retinal pigment epithelium RP (PPRPE), pigmented paravenous retinochoroidal atrophy, and Usher syndrome. The classification was based on previously published methods and rationale. ${ }^{15-1 \times}$ (Pigmented paravenous retinochoroidal degeneration has never been proved to be a hereditary disease but is probably a pattern of degeneration. Whether it should be classified as a form of retinitis pigmentosa is debatable. These patients were included in the RP section because their clinical and functional testing gave similar results to those of many patients with simplex RP. See Heckenlively and Kokame ${ }^{i x}$.) The designation of cone-rod degeneration was given if there was either electroretinographic evidence that the photopic electroretinogram was worse affected than the rod-isolated electroretinogram or that the final rod threshold was elevated less than 2.0 log units anywhere outside the macular area. Rod-cone degeneration was diagnosed if a patient's scotopic electroretinogram was more abnormal than the photopic waveform or if the final rod threshold was elevated, typically $3.4 \log$ units or more. In advanced cases of RP in which the visual field is less than $8^{\circ}$ it may not be possible to state whether a patient has the rod-cone or cone-rod pattern of degeneration on the basis of the dark adaptation test alone.

The study was performed in a masked fashion such that the investigators evaluating the fluorescein angiograms had no knowledge of the immunological activity results and vice versa. Detailed methodology for our evaluation of antiretinal antibodies and lymphocyte sensitisation to retinal antigens has been previously published" and will be described briefly. Sera from patients with RP and other types of ocular 


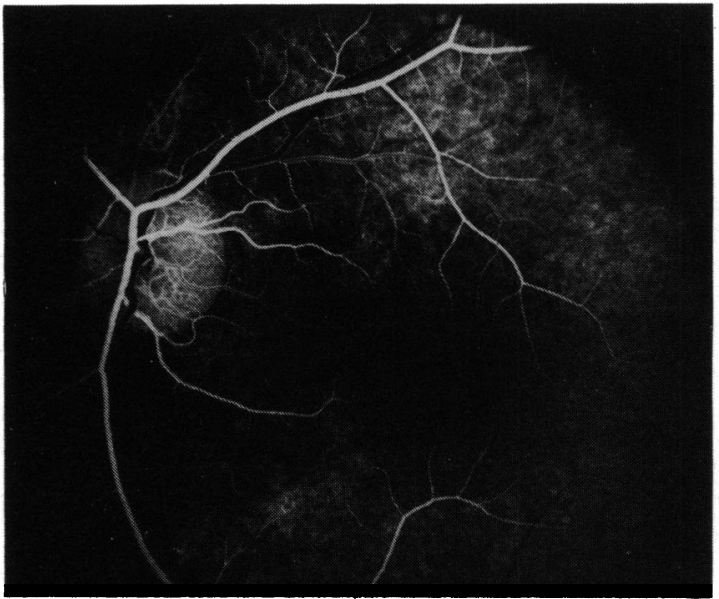

Fig. 1 Disc telangiectasia, mainly of temporal portion of disc, in 12-year-old boy with progressive cone-rod degeneration. Telangiectasia is most easily seen in early phases of the fluorescein angiogram.

disease were placed on normal human donor retinal sections, which were then washed and stained with fluorescein-conjugated goat antihuman IgG and IgM reagents. The sections were examined under a fluorescein microscope and graded for presence or absence of staining. Positive staining indicated the presence of IgG and/or IgM antigens in the patient's serum that bound antigens present on the sections. Peripheral blood lymphocytes obtained as previously

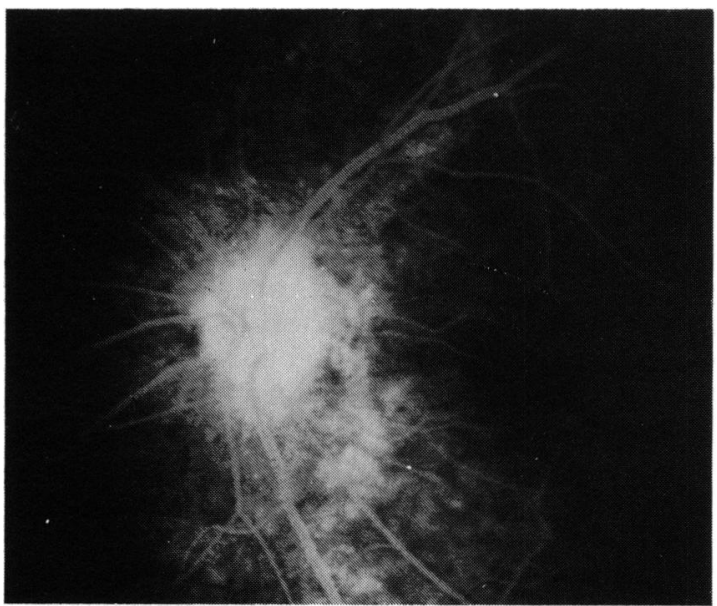

Fig. 2 Disc oedema, identified on late phases of the fluorescein angiogram, is seen in this patient with severe pigmented paravenous retinochoroidal atrophy (not shown). Leakage offluorescein causes the disc to be hyperfluorescent, obscuring disc detail, and may even leak into the overlying vitreous space.

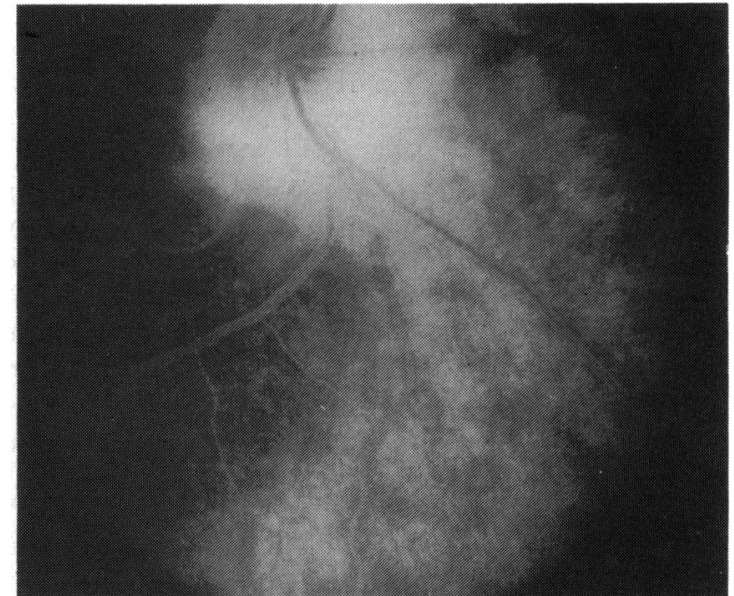

Fig. 3 Peripapillary oedema in a 20-year-old woman with Usher's syndrome. Hyperfluorescence in the peripapillary region obscures disc margin detail as well as most of the peripapillary retinal vessels. Diffuse retinal oedema is also present.

described" were incubated with T and B lymphocyte mitogens or retinal antigens. Tritiated thymidine was added four hours before harvesting. Sensitisation was expressed as a stimulation index, consisting of a ratio of counts per minute in the presence of antigen divided by counts per minute in the absence of antigen. A stimulation index $>2.0$ was considered an indication of cell-mediated activity against retinal antigen. Lymphocytes obtained from normal volunteers were used for controls for each method.

Six angiographic parameters were examined by two investigators: disc telangiectasia, disc staining, peripapillary oedema, arcade oedema, macular oedema, and focal vascular staining. The fluorescein angiographic parameters were scored (1) not present, (2) moderately present, and (3) markedly present. For statistical analysis the latter two groups were combined.

Disc telangiectasia, or dilatation of normal disc surface vessels, has been noted by us in a number of retinal dystrophies, in particular the cone-rod degenerations. ${ }^{19}$ These vessels can be identified by abnormal beading of the vessel walls and by the increased numbers of small vessels seen in the early phases of the fluorescein angiogram (Fig. 1).

Disc staining was evaluated on the late phases of the fluorescein angiogram; a positive finding was a brightly hyperfluorescent disc, often with overlying vitreal haze where fluorescein had escaped into the adjacent vitreous (Fig. 2).

Peripapillary oedema, evaluated on the late phases of the fluorescein angiogram, consisted or markedly hyperfluorescent areas covering at least five clock 


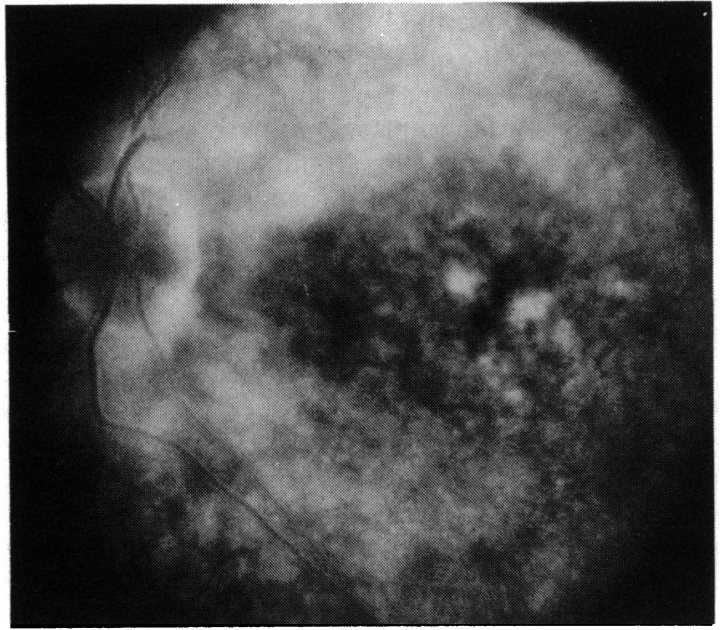

Fig. 4 Arcade oedema superiorly is particularly marked, though the retinal arcade inferiorly also shows

hyperfluorescence. Some macular and peripapillary oedema is also present. Patient is a 35-year-old woman with advanced simplex retinitis pigmentosa.

hours in the retina adjacent to the disc margin (Fig. 3).

Arcade oedema, evaluated on the late phases of the fluorescein angiogram, was identified when hyperfluorescent areas were noted deep to and adjacent to the vascular arcades of the posterior pole (Fig. 4). No attempt was made to distinguish whether the leakage was derived from retinal vasculature or retinal pigment epithelium.

Macular oedema was evaluated on late phases of the fluorescein angiogram and consisted of either the

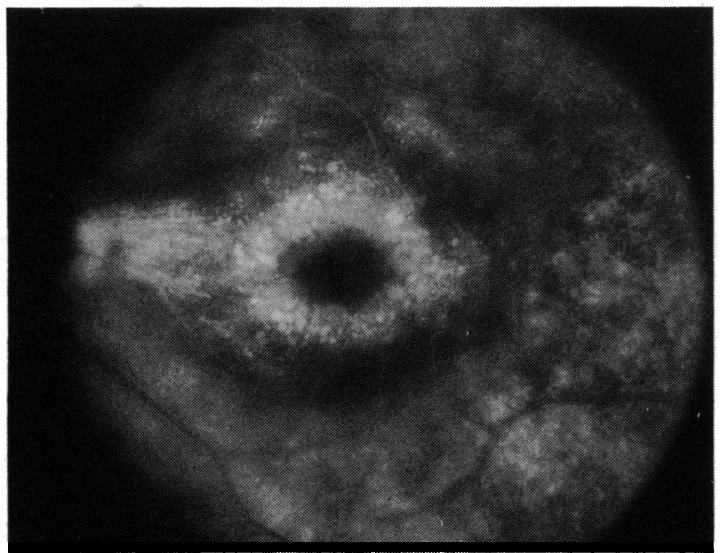

Fig. 5 Cystoid macular oedema, more in the paramacular region, is noted in this 43-year-woman with advanced simplex retinitis pigmentosa. Diffuse retinal oedema in the superior arcade and marked oedema of the maculopapillary bundle can be seen.

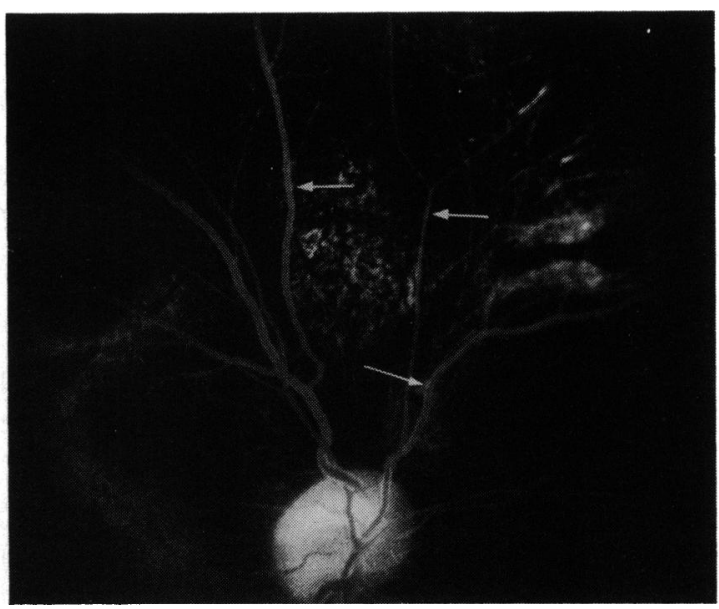

Fig. 6 Focal vascular staining consisting of focal areas of hyperfluorescence is seen in peripapillary retinal vessels as well as retinal veins and arteries superiorly (arrows) in a 23year-old man with choroideraemia.

traditional changes of cystoid macular oedema or diffuse hyperfluorescence of the posterior pole in the anatomical macular area (Fig. 5).

Vascular staining was noted on late phases of the fluorescein angiogram as focal, often pinpoint, areas of hyperfluorescence which could be seen in venous and arteriolar vessel walls (Fig. 6). Vascular staining was most easily seen in arcade and peripapillary area vessels (Fig. 7). At least two distinct focal areas of hyperfluorescence in a vessel wall were needed to call this parameter moderately present.

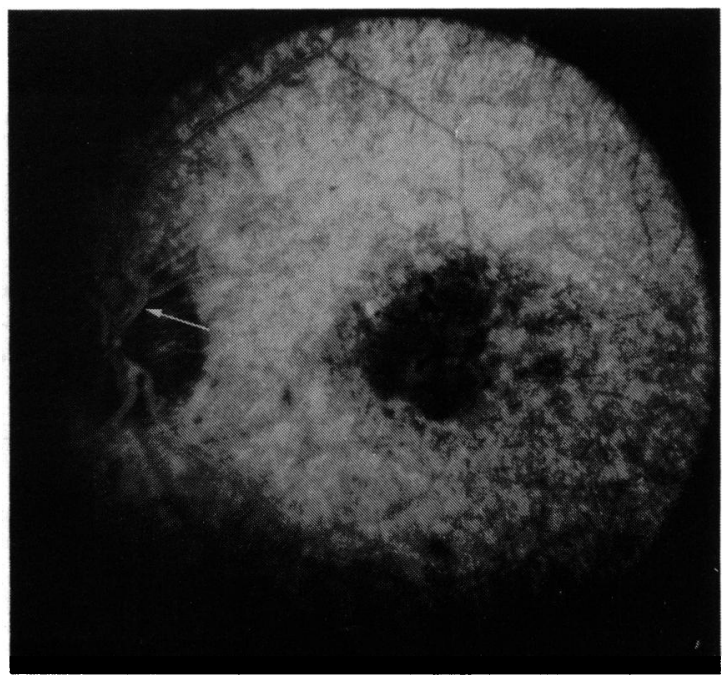

Fig. 7 Focal vascular staining in peripapillary retinal arteries (arrow), left eye, in a 42-year-old woman with advanced simplex retinitis pigmentosa. Severe diffuse retinal oedema is also present. 
An evaluation of the statistical association between fluorescein findings and immunological indices was performed by the one-tail Fisher's exact test. Group comparisons, such as RP with non-RP and rod-cone with cone-rod degeneration, were made by the Mantel-Haenszel test for homogeneity of $\chi^{2}$.

\section{Results}

Numerous significant correlations were found for all patients and for non-RP patients as a group. The nonRP disease group showed more significant correlations than the patients with RP.

For all patients (Table 1) positive immunofluorescence for IgG antiretinal antibodies correlated significantly with macular oedema $(\mathrm{p}<0.038)$ and disc staining $(p<0.033)$. A less significant correlation was noted between focal vascular staining $(p<0.068)$ and presence of $\mathrm{IgG}$, and between the lymphocyte stimulation test and macular oedema $(p<0 \cdot 079)$.

In an attempt to locate a cumulative effect, com-

Table 1 Correlation of immune reactivity with fluorescein angiographic parameters for all patients ( $R P$ and non- $R P$ )

\begin{tabular}{|c|c|c|}
\hline $\begin{array}{l}\text { Index of immune } \\
\text { reactivity }\end{array}$ & Angiographic change & $\begin{array}{l}\text { Significance } \\
\text { ( } p \text { value): } \\
\text { Fisher's exact } \\
\text { test }\end{array}$ \\
\hline IgG antiretinal antibody & Macular oedema & $<0.038$ \\
\hline IgG antiretinal antibody & Disc staining & $<0.033$ \\
\hline IgG antiretinal antibody & Focal vascular staining & $<0.068$ \\
\hline $\begin{array}{l}\text { Lymphocyte stimulation } \\
\text { (to retinal antigens) }\end{array}$ & Macular oedema & $<0.079$ \\
\hline $\begin{array}{l}\text { IgG or IgM antiretinal } \\
\text { antibodies }\end{array}$ & Disc staining & $<0.053$ \\
\hline $\begin{array}{l}\text { IgG, IgM antiretinal } \\
\text { antibodies and } \\
\text { lymphocyte } \\
\text { stimulation }\end{array}$ & Focal vascular staining & $<0.070$ \\
\hline
\end{tabular}

binations of immunoglobulin activity and/or lymphocycte stimulation were tested for significance; IgG or IgM antibody staining correlated with disc staining $(\mathrm{p}<0.05)$. Combinations of positive IgG and IgM staining, and lymphocyte stimulation tests, resulted in some correlation with focal vascular staining $(\mathrm{p}<0.07)$.

A number of significant correlations were found in the non-RP disease group (Table 2). IgG antiretinal antibody correlated with arcade oedema $(p<0.018)$, with disc staining $(\mathrm{p}<0.018)$, and with peripapillary oedema $(p<0.023)$. IgM staining correlated with peripapillary oedema $(p<0 \cdot 011)$. For a cumulative effect both IgG and IgM correlated with arcade oedema $(p<0.023)$, disc staining $(p<0.029)$, and peripapillary oedema $(p<0.003)$. Positive IgG, IgM, and lymphocyte stimulation tests together did not correlate significantly with any angiographic parameter. Attempts to subdivide the non-RP group between inflammatory and degenerative causes did not give significant results.

RP patients as a group had fewer correlations. IgG immonofluorescence correlated with arcade oedema $(\mathrm{p}<0.045)$, as did the combination fo IgG and IgM antibodies and positive lymphocyte stimulation $(\mathrm{p}<0 \cdot 045)$.

When the RP and non-RP groups were compared with each other, numerous significant differences appeared in correlations with arcade oedema and papillary oedema (Table 2 ).

RP patients were further compared by type of degeneration, that is, rod-cone and cone-rod, and were evaluated by analysis of variance with the nonRP patients (Table 3 ). The findings from the rodcone degeneration patients were significant or nearly so in correlations with arcade oedema. The findings from the cone-rod degeneration patients correlated nearly significantly in test correlations with focal vascular staining. No correlation was found between specific RP type and autoimmune reactivity.

Table 2 Correlation of immune reactivity with fluorescein angiographic parameters by groups ( $R P$ and non- $R P$ )

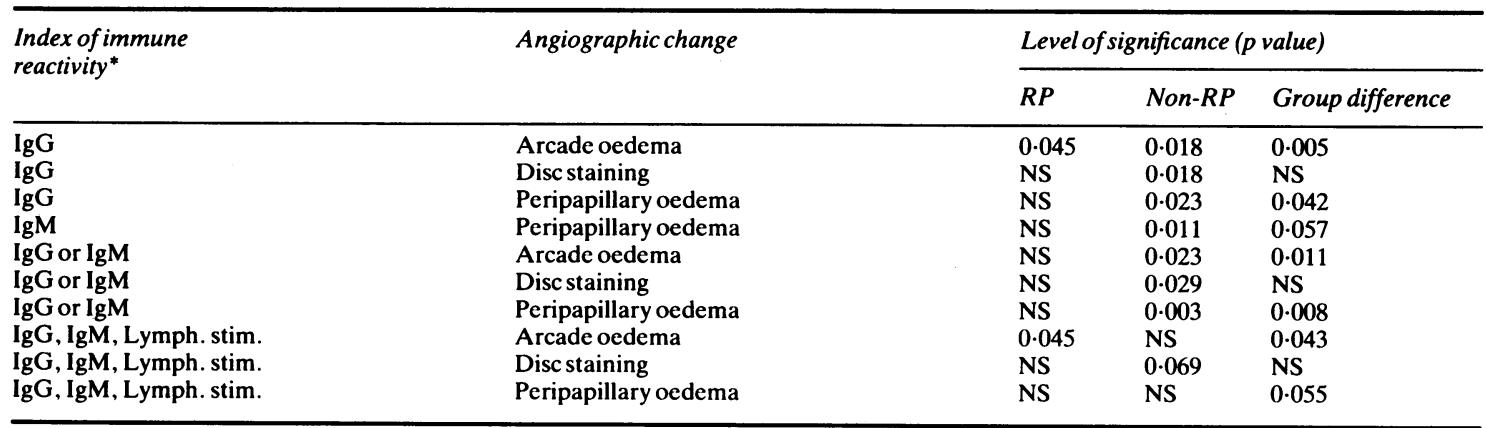

*IgG/IgM=positive IgG/IgM immunoglobulin indirect immunofluorescence. Lymph. stim. =lymphocyte stimulation by retinal antigens. NS=non-significant. 
Table 3 Correlation of immune reactivity with fluorescein angiographic parameters by retinitis pigmentosa degenerative type

\begin{tabular}{|c|c|c|c|}
\hline \multirow{2}{*}{$\begin{array}{l}\text { Index of immune } \\
\text { reactivity* }\end{array}$} & \multirow[t]{2}{*}{ Angiographic change } & \multicolumn{2}{|c|}{ Level of significance ( $p$ value) } \\
\hline & & Rod-cone & Cone-rod \\
\hline $\begin{array}{l}\text { IgG } \\
\text { Lymph. stim. } \\
\text { IgG, IgM, lymph. stim. } \\
\text { IgG, IgM, lymph. stim. }\end{array}$ & $\begin{array}{l}\text { Focal vascular staining } \\
\text { Arcade oedema } \\
\text { Arcade oedema } \\
\text { Focal vascular staining }\end{array}$ & $\begin{array}{l}\text { NS } \\
0 \cdot 067 \\
0 \cdot 011 \\
\text { NS }\end{array}$ & $\begin{array}{l}0.063 \\
\text { NS } \\
\text { NS } \\
0.069\end{array}$ \\
\hline
\end{tabular}

${ }^{*} \mathrm{IgG} / \mathrm{IgM}=$ positive IgG/IgM immunolglobulin indirect immunofluorescence. Lymph. stim. =lymphocyte stimulation by retinal antigens. NS=non-significant.

\section{Discussion}

While this study was originally designed to investigate the potential pathological role of the immune system in RP patients, the most significant results were found in the non-RP ocular disease reference group, which consisted mainly of other types of retinal disease (Table 4). These non-RP patients showed significant correlations between IgG and the combination of IgG and IgM antiretinal antibodies with arcade oedema, disc staining, and peripapillary oedema. IgM staining correlated significantly with peripapillary oedema.

RP patients as a group showed significant correlations between IgG alone and IgG, IgM antiretinal antibodies and lymphocyte stimulation with arcade oedema; when divided into rod-cone and cone-rod degeneration, neither group showed significant correlations singly with any immune index. Only the rod-cone degeneration group showed a significant correlation between the combination of IgG, IgM antiretinal antibodies and the lymphocyte stimulation test together as a cumulative effect with arcade oedema.

The existence of correlations between fluorescein angiographic changes and indices of immune activity may be an indication that the immune system has a pathophysiological role in a number of retinal

Table 4 Diagnoses of patients with non-RP ocular disease

Birdshot retinochoroiditis

Cataract with macular degencration

Cataract with retinal detachment

Detached retina

Diabetic retinopathy

Fundus flavimaculatus

High myopia

Macular degeneration (age related)

Macular oedema (after cataract surgery)

Measles retinopathy

Regional choroidal atrophy

Secondary luetic chorioretinitis

Stickler syndrome

Toxoplasmosis

Uveitis diseases. This correlation does not clarify whether the observed changes represent a primary pathological process, a secondary effect in a complicated degenerative process, or possibly some type of epiphenomenon. The observation that RP patients show fewer such correlations than non-RP patients may not reflect a lack of immunological activity but rather that the slow defenerative process inherent in RP does not challenge the immune system in the same fashion as the process of inflammatory disease. Eye tissue compared with total body mass is small to begin with, and products from a retinal degeneration would not be a large antigenic challenge.

Fluorescein angiography is commonly used for investigating various types of retinal oedema and less commonly for disc telangiectasia. Focal vascular staining on late phases of the fluorescein angiogram is a finding not commonly associated with hereditary retinal degenerations, or even other retinal diseases except vasculitis. The cause of focal vascular staining is unknown, but it could be related to the presence of immune complexes or other immunological entity producing damage to retinal vessels.

Peripapillary oedema was significantly associated with both presence of antiretinal antibodies and lymphocyte sensitisation. Since the peripapillary area, particularly when a conus is present, tends to be unprotected by the blood-retinal and retinal pigment epithelial barriers, it is not surprising that antiretinal immunological mechanisms might damage this area.

The correlations between the fluorescein angiogram and markers of immune reactivity in this study lead us to assume that they reflect secondary rather than primary disease effects and that the pathological influence of immune factors remains to be determined. Indirect immunofluorescent staining with the use of patient sera on human donor tissue has proved valuable in detecting antiretinal antibodies, and it merits further investigation for its development as a possible clinical tool.

We thank Professor Alan Bird for revicwing the manuscript and Mrs Mada Barrett for editorial assistance. 
This rescarch was supported by a centre grant from the National Retinitis Pigmentosa Foundation, a grant-in-aid from the National Socicty to Prevent Blindness (SMC), and USPHS grants EY 1309 (RME) and EY 00331 from the National Eyc Institute.

\section{References}

1 Dumonde DC, Kasp-Grochowska E, Graham E, Sanders MD. Anti-retinal autoimmunity and circulating immune complexes in patients with retinal vasculitis. Lancet 1982 ; ii: 787-92.

2 Keltner JL, Roth AM, Chang RS. Photoreceptor degeneration: possible autoimmune disorder. Arch Ophthalmol 1983; 101: 564-5.

3 Brinkman CJJ, Pinckers JLG, Brockhuyse RM. Immunc reactivity to different retinal antigens in patients suffering from retinitis pigmentosa. Invest Ophthalmol Vis Sci 1980; 19: 743-50.

4 Heredia CD, Vich JM, Huguet J, Garcia-Calderon JV, GarciaCalderon PA. Altered cellular immunity and suppressor ccll activity in patients with primary retinitis pigmentosa. $\mathrm{Br} J$ Ophthalmol 1981; 65: 850-4.

5 Char DH, Bergsma DR, Rabson AS, Albert DM, Herberman RB. Cell-mediated immunity to retinal antigens in patients with pigmentary retinal degenerations. Invest Ophthalmol Vis Sci 1974; 13: 198-203.

6 Newsome DA, Nussenblatt RB. Retinal S antigen reactivity in patients with retinitis pigmentosa and Usher's syndrome. Retina 1984; 4: 195-9.

7 Nussenblatt RB, Mittal KK, Ryan S, Green WR, Maumenec AE. Birdshot retinochoroidopathy associated with HLA-A29 antigen and immune responsiveness to retinal S-antigen. $\mathrm{Am} \mathrm{J}$ Ophthalmol 1982; 94: 147-58.
8 Nussenblatt RB, Gery I, Ballintinc E. Cellular immunc responsiveness of uveitis patients to retinal S-antigen. Am J Ophthalmol 1980; 89: 173-9.

9 Hooks JJ, Detrick-Hooks B, Geis S, Newsome DA. Retinitis pigmentosa associated with a defect in the production of interferon-gamma. Am J Ophthalmol 1983; 96: 755-8.

10 Rahi AHS. Autoimmunity and the retina. II. Raised scrum IgM levels in retinitis pigmentosa. Br J Ophthalmol 1973; 57: 904-9.

11 Spiro R, Weleber R, Kimberling W. Serum IgM in retinitis pigmentosa: a genetic study. Clin Genet 1978; 13: 295-304.

12 Kumar M, Gupta RM, Nema HV. Role of autoimmunity in retinitis pigmentosa. Ann Ophthalmol 1983; 15: 838-40.

13 Spalton DJ, Rahi AHS, Bird AC. Immunologic studics in retinitis pigmentosa associated with retinal vascular leakage. $\mathrm{BrJ}$ Ophthalmol 1978; 62: 183-7.

14 Chant SM, Heckenlively JR, Mcyers-Elliott RH. Autoimmunity in hereditary retinal degencration. I. Basic studics. $\mathrm{Br} J$ Ophthalmol in press.

15 Krill AE. Hereditary retinal and choroidal diseases. Hagerstown: Harper and Row, 1977.

16 Heckenlively JR. Preserved para-arteriolar retinal pigment cpithclium (PPRPE) in retinitis pigmentosa. Br J Ophthalmol 1982; 66: 26-30.

17 Heckenlively JR. The hereditary retinal degencrations. In: Emcry A, Rimoin D, cds. Principles and practice of medical genetics. Edinburgh: Churchill-Livingstonc, 1983; 522-38.

18 Heckenlively JR, Kokame G. Pigmented paravenous retinochoroidal degeneration. Doc Ophthalmol Proc Ser 1984; 40: 236-41.

19 Heckenlively JR, Martin DA, Rosales TO. Telangiectasia and optic atrophy in cone-degencrations. Arch Ophthalmol 1981;99. 1983-91. 